Cahiers $d u$ MONDE RUSSE

\section{Cahiers du monde russe}

Russie - Empire russe - Union soviétique et États indépendants

$44 / 4 \mid 2003$

Varia

\title{
Le conseil du tsar dans la culture politique de
}

l'époque pétrovienne

La genèse du Conseil suprême secret, fin XVIIe s.-1726

Anna Joukovskaïa-Lecerf

\section{OpenEdition}

Journals

Édition électronique

URL : https://journals.openedition.org/monderusse/8624

DOI : $10.4000 /$ monderusse. 8624

ISSN : $1777-5388$

Éditeur

Éditions de l'EHESS

Édition imprimée

Date de publication : 1 octobre 2003

Pagination : 577-604

ISBN : 2-7132-1833-0

ISSN : $1252-6576$

Référence électronique

Anna Joukovskaïa-Lecerf, « Le conseil du tsar dans la culture politique de l'époque pétrovienne ». Cahiers du monde russe [En ligne], 44/4 | 2003, mis en ligne le 01 janvier 2007, consulté le 02 septembre 2022. URL : http://journals.openedition.org/monderusse/8624 ; DOI : https://doi.org/ $10.4000 /$ monderusse. 8624 
chercher : repérer : avancer

Cet article est disponible en ligne à l'adresse :

http://www.cairn.info/article.php?ID REVUE=CMR\&ID NUMPUBLIE=CMR 444\&ID ARTICLE=CMR 4440577

Le conseil du tsar dans la culture politique de l'époque pétrovienne. La genèse du Conseil suprême secret, fin XVIle s. -1726

\title{
par Anna JOUKOVSKAÏA-LECERF
}

\section{| Editions de l'EHESS | Cahiers du monde russe}

\author{
2003/4 - Vol 44 \\ ISSN 1252-6576 | ISBN 2713218330 | pages 577 à 604
}

Pour citer cet article :

-JOUKOVSKAÏA-LECERF A., Le conseil du tsar dans la culture politique de l'époque pétrovienne. La genèse du Conseil suprême secret, fin XVlle s.-1726, Cahiers du monde russe 2003/4, Vol 44, p. 577-604.

Distribution électronique Cairn pour les Editions de l'EHESS.

(C) Editions de l'EHESS. Tous droits réservés pour tous pays.

La reproduction ou représentation de cet article, notamment par photocopie, n'est autorisée que dans les limites des conditions générales d'utilisation du site ou, le cas échéant, des conditions générales de la licence souscrite par votre établissement. Toute autre reproduction ou représentation, en tout ou partie, sous quelque forme et de quelque manière que ce soit, est interdite sauf accord préalable et écrit de l'éditeur, en dehors des cas prévus par la législation en vigueur en France. Il est précisé que son stockage dans une base de données est également interdit. 


\title{
LE CONSEIL DU TSAR DANS LA CULTURE POLITIQUE DE L'ÉPOQUE PÉTROVIENNE
}

\author{
La genèse du Conseil suprême secret, \\ fin $\mathrm{XVII}^{\mathrm{e}}$ s.-1726
}

Le mot conseil (sovet, duma) revient souvent dans les textes anciens russes portant sur le pouvoir. Il fut notamment utilisé pour évoquer des décisions individuelles ${ }^{1}$. Aujourd'hui singulier, cet usage témoigne que dans la conscience de l'homme médiéval toute prise de décision impliquait une discussion, un conseil préalable. Ainsi, la Rus' partagea-t-elle avec l'Europe occidentale «l'antique principe du gouvernement féodal : pas de décision ni d'intervention sans consultation $»^{2}$. Entre le $\mathrm{XI}^{\mathrm{e}}$ et le $\mathrm{XVI}^{\mathrm{e}}$ siècle, le thème de bon souverain chrétien qui agit dans la concorde avec ses sages conseillers fut décliné par les auteurs russes, dont des personnages éminents comme Maxime le Grec et Andrej Kurbskij, au point de devenir un topique ${ }^{3}$.

Cependant, ce modèle idéologique quasi universellement accepté ne donna pas naissance à une théorie spécifique relative à l'art du gouvernement. Aucune théorie

1. I. P. Kulakova, « Social’no-političeskaja terminologija i evoljucija vzaimootnošenij vlasti i soslovij v Rossii vtoroj poloviny XVI-načala XVII vv.» (La terminologie sociopolitique et l'évolution des rapports entre le pouvoir et les états en Russie durant la seconde moitié du XVI et le début du XVII ${ }^{\mathrm{e}}$ siècle), in Soslovija i gosudarstvennaja vlast' $v$ Rossii. $X V$-seredina XIX vV. Meždunarodnaja konferencija - čtenija pamjati akademika L. V. Čerepnina. Moskva, 13-16 ijunja $1994 \mathrm{~g}$. Tezisy dokladov (Les états et le pouvoir gouvernant en Russie, $x v^{e}$ mi-XIXe s.), Moscou, 1994, p. 264-272, ici p. 266.

2. Daniel Roche, La France des Lumières, Paris, Fayard, 1993, p. 134.

3. Sur ce sujet voir Sergei Bogatyrev, The sovereign and his counsellors : ritualised consultations in Muscovite political culture, 1350s-1570s, Helsinki, Academia Scientiarum Fennica, 2000, p. 37-98 (version électronique). À l'origine, le thème du souverain et de ses conseillers fut emprunté à la littérature byzantine. 
de cet ordre ne fut connue en Russie moscovite avant le début du XVIII ${ }^{\mathrm{e}}$ siècle, quand Pierre Irr fit la découverte, d'ailleurs superficielle, du caméralisme suédois ${ }^{4}$. Aucune tentative de conceptualiser ou de réglementer le fonctionnement réel du conseil du tsar ne nous est révélée par les sources conservées. À la limite, l'existence même en Russie moscovite d'un conseil du tsar officiel pose problème, malgré une longue tradition historiographique qui attribue le statut d'un conseil d'État doté du pouvoir consultatif à la Bližnjaja duma des XVI même à la Bojarskaja duma 5 . S'il est indéniable que la tradition des consultations ritualisées entre le tsar et les dignitaires de son choix fut très ancienne et très vivace, rien n'indique qu'elle ait été institutionnalisée ; et si les dignitaires choisis jouèrent effectivement le rôle de conseillers intimes du tsar, ils n'en eurent pas le statut. Un tel statut n'avait pas été défini dans la culture politique de la Russie moscovite ${ }^{6}$.

Ce fond historique donne du relief aux événements du début de 1726 dont l'interprétation fait l'objet du présent article. En février apparut le premier conseil du tsar formellement institué de l'histoire russe, le Conseil suprême secret ${ }^{7}$. Fin mars l'impératrice approuva un Avis sur le nouveau Conseil secret qui est institué ${ }^{8}$, document dans lequel les membres du Conseil firent une tentative sans précédent en Russie de définir le conseil du tsar: décrire ses attributions, sa position par rapport au souverain et aux institutions centrales, la procédure à y respecter.

Le contexte politique de la création du Conseil suprême secret ainsi que ses activités jusqu'en 1730, date de sa disparition, ont été beaucoup étudiés ${ }^{9}$. Par contre, le

4. Claes Peterson, Peter the Great's administrative and judicial reforms : Swedish antecedents and the process of reception, Stockholm, Nordiska bokhandeln, 1979.

5. Voir une analyse de cette tradition historiographique dans Sergei Bogatyrev, op. cit., Appendix I, p. 223-260.

6. Ibid., p. 90-91. Après Talcott Parsons, Bogatyrev entend par rôle les actions qu'une personne accomplit dans ses relations avec les autres et qui sont évaluées sous l'angle de leur signification fonctionnelle dans un système social, et par statut la localisation d'une personne dans le système social par rapport aux autres acteurs (T. Parsons, The social system, Londres, Routledge, 1991, nouvelle édition, p. 25). Nous utiliserons ici la même définition.

7. Verhovnyj tajnyj sovet. Nous traduisons littéralement l'expression russe tajnyj sovet par conseil secret plutôt que par conseil privé, plus habituel. Il nous paraît important de distinguer entre les adjectifs tajnyj (secret) et bližnij (privé, intime), surtout quand ils font partie de noms d'institutions : Verhovnyj tajnyj sovet, mais Bližnjaja duma. Tajnyj insiste sur le fait que les affaires traitées au conseil doivent rester sous secret, tandis que bližnij souligne l'intimité des relations entre les conseillers et le tsar.

8. Mnenie v ukaz o novom učreždennom Tajnom sovete. Le texte est publié dans Sbornik imperatorskogo russkogo istoričeskogo obščestva (Recueil de la société impériale de l'histoire de la Russie, cité infra SIRIO), Saint-Pétersbourg, vol. 55, p. 93-97.

9. Une bibliographie quasi exhaustive des ouvrages parus en langue russe jusqu'en 1996 est fournie par M. V. Babič, Gosudarstvennye učreždenija XVIII veka (Les institutions d'État au XVIII ${ }^{e}$ s.), Moscou, 1999. Voir aussi John LeDonne, «Ruling families in the Russian political order », Cahiers du Monde russe et soviétique, 28/3-4, juillet-décembre 1987, p. 233-322 ; E. V. Anisimov, Gosudarstvennye preobrazovanija i samoderžavie Petra Velikogo v pervoj četverti XVIII veka (Les réformes d'État et l'autocratie de Pierre le Grand durant le premier quart du XVIII siècle), Saint-Pétersbourg, Dmitrij Bulanin, 1997, p. 231-239 ; A. B. Kamenskij, Ot Petra I do Pavla I. Reformy v Rossii XVIII veka. Opyt celostnogo analiza (De Pierre Ier à Paul I'r. Les réformes en Russie au XVIII siècle. Essai d'analyse globale), Moscou, Rossijskij gosudarstvennyj gumanitarnyj universitet, 2001,p. 184-218. 
processus de gestation de l'idée même de créer un conseil institutionnalisé et réglé auprès du monarque russe et d'introduire dans la hiérarchie politique le statut de conseiller du tsar n'a pas été spécialement analysé, ainsi que les formes particulières que prit par conséquent l'organisation initiale du Conseil suprême secret. Remplir cette lacune, à travers notamment une étude détaillée de la genèse de l'Avis sur le nouveau Conseil secret, est le but des pages qui suivent.

Rappelons brièvement les événements de 1725-1726 liés à la formation du Conseil suprême secret. Sa création fut proclamée un an après la mort de Pierre Ier, dans une situation politique proche d'un interrègne. L'impéritie de Catherine Ire l'exigeait, aucun des principaux ministres n'étant alors suffisamment puissant pour s'imposer comme tuteur unique de l'impératrice. On sait en effet que la nécessité de se réunir pour se concerter était apparue aux ministres aussitôt après l'avènement de Catherine Ire. Courant 1725, il se forma autour d'elle un cercle restreint de sept personnes qui la conseillaient de façon informelle ${ }^{10}$. Il s'agissait du chef de l'armée Aleksandr Menšikov, du chef de la marine Fedor Apraksin, du chef de la diplomatie Gavriil Golovkin, de son adjoint Andrej Osterman, des sénateurs Petr Tolstoj et Dmitrij Golicyn, et du procureur général du Sénat, Pavel Jagužinskij ${ }^{11}$. Les réunions, que leurs participants évoquaient sous le nom de conseils secrets, ne laissèrent pas de traces écrites ; elles se tenaient soit chez l'impératrice ${ }^{12}$, soit dans les locaux du collège des Affaires étrangères ${ }^{13}$.

On ignore le nom de la personne qui suggéra de donner à ce cercle de dignitaires le statut d'un conseil du tsar officiel. Toujours est-il que l'impératrice jugea l'idée bonne. Le 8 février 1726, elle signa un oukase sur l'instauration, sous sa propre présidence, du Conseil suprême secret « pour les affaires d'État importantes, extérieures et intérieures $»^{14}$. Les six personnages mentionnés constituèrent l'effectif initial du Conseil suprême secret (Jagužinskij, ennemi juré de Menšikov, fut le seul exclu parmi les participants des conseils secrets de 1725). Tous les membres portaient le grade de conseiller secret actuel (dejstvitel'nyj tajnyj sovetnik).

Le choix du nom pour le Conseil ne fut pas fortuit. Si l'adjectif suprême était une innovation dans le vocabulaire politique et administratif (il servait à souligner que le Conseil dominait le Sénat), l'expression conseil secret avait eu une longue histoire qui servira de fil à notre exposé.

10. Certains diplomates étrangers le signalaient dans leurs dépêches, voir $S I R I O$, vol. 3, p. 409 (Lefort), vol. 15,p. 274 (Mardefeld) et vol. 64,p. 46, 60 et 92 (Campredon). En outre, une indication directe sur cette pratique se trouve dans le texte de l'oukase de Catherine Ire du 8 février 1726 concernant l'institution du Conseil suprême secret, voir Polnoe sobranie zakonov Rossijskoj Imperii (Collection complète des lois de l'Empire Russe, cité infra PSZ), Saint-Pétersbourg, 1830, vol. 7, no 4830, p. 568-569.

11. Cette composition est indiquée dans un procès-verbal du Conseil suprême secret du 9 février 1726, qui résume l'histoire de son instauration, Voir SIRIO, vol. 55, p. 1.

12. S. M. Solov'ev, Istorija Rossii s drevnejših vremen (Histoire de la Russie depuis l'Antiquité), Moscou, Mysl', 1963, vol. 9, p. 618.

13. SIRIO, vol. 55 , p. 1.

14. PSZ, vol. 7, no 4830, p. 568-569. 


\section{Le conseiller du tsar : un rôle et un statut}

Durant la dernière décennie du XVII siècle, le délabrement de l'ancienne hiérarchie des grades moscovites devint manifeste. Pierre $\mathrm{I}^{\mathrm{er}}$ accordait de plus en plus rarement les grades de bojarin ou d'okol'ničij leur préférant les grades militaires et civils d'origine étrangère, qu'il introduisait d'ailleurs cas par cas, sans système apparent (plusieurs tombèrent en désuétude sous son règne ou peu après). La situation se stabilisa seulement en 1722, avec la promulgation de la Table des rangs. Entre-temps, les dignitaires du tsar avaient peu de repères sûrs, formels, pour se situer les uns par rapport aux autres. Pierre ${ }^{\mathrm{I} r}$ se préoccupait peu des problèmes psychologiques et sociaux que créait cette situation, assez pénible sans doute pour les membres de l'élite gouvernante : leur monde représentait une échelle, et c'était à peine si deux familles pouvaient coexister paisiblement au même échelon ${ }^{15}$. Le déclin des grades moscovites n'était d'ailleurs qu'un signe des changements plus profonds qui s'accomplirent dans les premières années du XVIII ${ }^{\mathrm{e}}$ siècle : le démantèlement progressif de la cour du souverain (Gosudarev dvor) et l'étiolement de la Bližnjaja duma ${ }^{16}$.

Il n'est pas surprenant de voir que, au moment où l'ancienne tradition des consultations ritualisées entre le monarque et ses dignitaires choisis perdait visiblement la netteté des formes organisationnelles qu'elle avait acquises durant son histoire pluriséculaire ${ }^{17}$, certains membres de l'élite gouvernante s'intéressèrent au statut des conseillers des autres monarques européens ${ }^{18}$. Ainsi le bojarin Andrej Matveev, ambassadeur russe à Paris en 1705-1706 et à Londres en 1706, témoigna d'un vif intérêt pour les conseils du roi de France et de la reine d'Angleterre. Lors de son séjour en France, il tint un journal fort intéressant ${ }^{19}$, dans lequel la description du gouvernement central occupe une place importante. Matveev recueillit notamment des informations assez exactes sur la composition, les compétences et la procédure du Conseil du roi. Il nota avec précision les fonctions des ministres et des secrétaires d'État tout en soulignant que le Conseil ne limitait aucunement le pouvoir du souverain : le roi de France était un monarque absolu. Un extrait de ce journal, fait par Matveev lui-même et intitulé Une brève description des grades, ou des rangs, de la cour de France $[. . .]^{20}$, fut envoyé à Pierre $\mathrm{I}^{\mathrm{er}}$ en mai

15. Sur ce sujet voir André Berelowitch, La hiérarchie des égaux. La noblesse russe d'Ancien Régime, $X V I^{e}-X V I I^{e}$ S., Paris, Seuil, 2001.

16. E. V. Anisimov, op. cit., p. 18-41.

17. Sur l'évolution de ses formes voir Sergei Bogatyrev, op. cit., p. 99-222.

18. Hypothèse judicieuse exprimée par I. S. Šarkova et A. D. Ljublinskaja, «A. A. Matveev i ego trud» (A. A. Matveev et son œuvre), in id., eds, Russkij diplomat vo Francii (Zapiski Andreja Matveeva) (Un diplomate russe en France. Le journal d'Andrej Matveev), Leningrad, Nauka, 1972, p. 21-22.

19. Analysé par Wladimir Berelowitch, « Aux sources d'un modèle à construire : la France de 1705 vue par un Russe », in Martine Godet, ed., De Russie et d'ailleurs. Feux croisés sur l'histoire. Pour Marc Ferro, Paris, Institut d'études slaves, 1995, p. 389-403.

20. Opisanie korotkoe francuzskogo dvora činam ili rangam [...] : Rossijskij gosudarstvennyj arhiv drevnih aktov (Archives des actes anciens de l'État de Russie, cité infra RGADA), fond 370 , opis' 1 , delo 3,1. 1-9 ob. 
170921. Arrivé à Londres, Matveev composa deux mémoires concernant l'organisation du pouvoir central en Angleterre. Ils furent envoyés en Russie en juillet 170722. Le premier document contient une liste des principaux magistrats du roi Guillaume III selon les Statuts de 1692, et le deuxième une description circonstanciée du gouvernement de la reine Anne Stuart, soit du Parlement et surtout du Conseil privé.

En juillet 1709, il se produisit en Russie un événement qui, sans qu'on puisse affirmer qu'il était un effet direct de l'extrait du journal de Matveev, prouvait que l'intérêt de son auteur pour le statut des conseillers royaux était partagé par ses pairs. Cet événement fut l'introduction de quatre nouvelles positions dans la hiérarchie des grades civils: chancelier, vice-chancelier, conseiller secret actuel et conseiller secret. Certains historiens pensent que les deux derniers grades n'étaient que « des titres honorifiques qui n'étaient associés directement ni à des fonctions ni à des devoirs concrets » ${ }^{23}$. Ces grades n'attachèrent en effet leurs porteurs à aucune institution, puisque Pierre Irr n'avait pas de conseil permanent. Toutefois, pour se prononcer sur la véritable portée de cet épisode, il faut se souvenir de son contexte historique et, si l'on peut dire, psychologique.

Le tsar institua ces quatre grades au lendemain de la victoire de Poltava ${ }^{24}$, qui détermina l'issue de la guerre du Nord et, en grande partie, toute l'histoire ultérieure du règne de Pierre $\mathrm{I}^{\mathrm{e} 25}$. Il fut pleinement conscient de toutes les implications politiques de cette victoire ${ }^{26}$. Le caractère extraordinaire des circonstances dans lesquelles Pierre Irr choisit d'attribuer les grades mentionnés à quatre de ses serviteurs nous permet d'apprécier la valeur que le tsar et les élus eux-mêmes devaient attacher à cette récompense. Outre cela, un coup d'œil sur les personnages des quatre titulaires fait découvrir la signification des nouveaux grades.

Gavriil Golovkin et Petr Šafirov, les codirecteurs du service diplomatique, furent nommés, respectivement, chancelier et vice-chancelier. Golovkin, lointain parent, ancien chambellan et « ministre intime » de Pierre Ier , était inséparable de lui. Šafirov, diplomate talentueux issu d'un milieu modeste ${ }^{27}$, fut le deuxième fruit des

21. S. M. Troickij, Russkij absoljutizm i dvorjanstvo v XVIII veke : formirovanie bjurokratii (L'absolutisme russe et la noblesse au XVIII siècle : la formation d'une bureaucratie), Moscou, Nauka, 1974,p. 54.

22. Ibid., p. 58.

23. L. E. Šepelev, Činovnyj mir Rossii, XVIII - načalo XIX veka (Le monde de la bureaucratie russe, $X V I I I^{e}$-début XIX ${ }^{\mathrm{e}}$ siècle), Saint-Pétersbourg, Iskusstvo-SPB, 1999, p. 134.

24. Pis'ma i bumagi Imperatora Petra Velikogo, 1709 (Lettres et papiers de l'empereur Pierre le Grand, 1709), Moscou, Izd.-vo AN SSSR, 1950, p. 243-264.

25. P. Englund, The battle of Poltava. The birth of the Russian Empire, Londres, V. Gollancz, 1992.

26. Les jugements personnels de Pierre I ${ }^{e r}$ à propos de la victoire de Poltava sont analysés avec finesse par E. A. Pogosjan, Petr I - arhitektor rossijskoj istorii (Pierre I ${ }^{e r}$, architecte de l'histoire russe), Saint-Pétersbourg, Iskusstvo-SPB, 2001, p. 112-124 et 17-19. Dans l'entourage de Pierre Ire , on pensait que, depuis cette victoire, le tsar pouvait prétendre au titre d'empereur, voir A. M. Pančenko, Russkaja kul'tura v kanun petrovskih reform (La culture russe à la veille des réformes de Pierre le Grand), Leningrad, Nauka, 1984, p. 121-123.

27. D. [O.] Serov, «Zametka o biografii Šafirova » (Note sur la biographie de Šafirov), Study Group on Eighteenth-Century Russia Newsletter, 21, 1993, p. 57-63. 
expériences méritocratiques du tsar (après le favori Menšikov). Le prince Grigorij Dolgorukij fut promu au grade de conseiller secret actuel. Diplomate éminent, il appartenait à une des plus illustres familles russes dans laquelle le grand-père de Pierre Ier avait choisi sa première femme. Enfin, le bojarin Ivan Musin-Puškin (haut dignitaire, parent et proche collaborateur de Pierre Ier) reçut le grade de conseiller secret.

Dolgorukij et Musin-Puškin exerçaient des fonctions différentes, mais partageaient le même privilège qui était aussi un devoir : depuis des années, ils restaient parmi les plus proches interlocuteurs de Pierre Ier. Les grades de conseiller secret actuel et de conseiller secret les distinguèrent nettement dans le cercle des personnes que le tsar consultait plus ou moins régulièrement. L'instauration de ces grades esquissa une séparation entre ceux qui jouaient le rôle de conseiller du tsar et ceux qui en avaient le statut. Est-ce une coïncidence si les premiers échos des discussions sur l'institution d'un conseil du tsar parvenus à nos jours datent de cette époque ?

\section{Les projets de Heinrich Fick : un absolutisme assisté}

Réunir autour de lui, lors de ses déplacements innombrables ou de ses courts séjours dans la capitale, tantôt des généraux pour discuter des problèmes militaires, tantôt des diplomates pour leur parler politique, fut une méthode de prise de décision habituelle pour Pierre $\mathrm{I}^{\mathrm{r} 28}$. Mais ce fut à partir de 1710 seulement que les commis de la chancellerie des Ambassades (Posol'skaja kanceljarija) utilisèrent l'expression conseil secret à propos des consultations sur les affaires diplomatiques que Golovkin, Šafirov, Dolgorukij et le conseiller secret Petr Tolstoj menaient en présence du tsar ou sans lui. Rien ne permet de penser cependant qu'il s'agissait d'un conseil permanent légalement établi. Il faut croire que les commis suivaient simplement les desiderata de leurs chefs ${ }^{29}$.

En février 1711, avant de partir sur le théâtre des opérations militaires contre la Porte Ottomane, Pierre Ir nomma un comité de neuf personnes, le Sénat, pour diriger le pays en son absence ${ }^{30}$. Le chancelier et le vice-chancelier qui devaient partir avec le tsar n'y étaient pas inclus. Cela signifiait que le Sénat n'était pas censé intervenir dans la gestion des affaires étrangères ${ }^{31}$. Pendant cette campagne, toutes les questions diplomatiques importantes (conclusion d'une alliance avec le hospodar de Moldavie, conditions de paix avec la Porte Ottomane) étaient discutées

28. Voir, par exemple, E. P. Pod'japol'skaja, «Voennye sovety 1708-1709 godov» (Les conseils de guerre de 1708-1709), in L. G. Beskrovnyj et al., eds, Poltava. K 250-letiju Poltavskogo sraženija (Poltava. À l'occasion du 250 anniversaire de la bataille de Poltava), Moscou, 1959, p. 112-136.

29. Sur ces réunions, voir notre article « À propos de la création du collège des Affaires étrangères sous Pierre le Grand », Cahiers du Monde russe, 43/1, janvier-mars 2002, p. 57-66.

30. Oukases du 22 février et du 2 et du 5 mars 1711 : N. A. Voskresenskij, ed., Zakonodatel'nye akty Petra I (Actes législatifs de Pierre I ${ }^{\text {er }}$, cité infra ZA) Moscou-Leningrad, Akademija Nauk SSSR, 1945, nos 240-244,p. 197-204.

31. L'oukase de 2 mars 1711, où sont énumérées ses responsabilités, le confirme : Ibid., n 241 , p. $198-200$. 
et résolues dans des consultations entre le tsar, le chancelier et le vice-chancelier, ainsi que des diplomates et des généraux dont certains portaient les titres de conseillers secrets ou de conseillers secrets actuels ${ }^{32}$. Ainsi, dès 1711 , commença à se dessiner dans le gouvernement russe une dichotomie nouvelle : le Sénat pour les affaires intérieures / les conseils secrets pour les affaires extérieures.

Toutefois, en 1715, l'appellation conseil secret fut abandonnée pour collège secret et, à partir de 1716, pour collège des Affaires étrangères, ou collège des Ambassades $^{33}$. Loin d'être fortuits, ces changements suivaient les idées de Pierre Ier, lui-même influencé par la lecture d'un mémoire de Heinrich Fick consacré à l'introduction des collèges en Russie ${ }^{34}$. Allemand en service auprès du tsar depuis 1714 , Fick fut l'un des principaux responsables de la célèbre réforme des institutions centrales menée en 1717-172235.

Dans son premier mémoire ${ }^{36}$, Fick proposait l'établissement de sept collèges présidés chacun par un sénateur : le collège de la Justice (ou le Haut sénat), le collège de la Chancellerie (ou la Grande chancellerie), le collège de l'Amirauté, ceux de la Guerre, des Revenus, du Trésor et du Commerce. Les Affaires étrangères manquaient dans cette liste. D'après l'idée de Fick, elles devaient entrer dans la sphère de compétence du collège de la Chancellerie, qui travaillerait en contact beaucoup plus étroit avec le tsar que les autres collèges : il préparerait et expédierait « tous les ordres de S.M. Tsarienne aux amiraux, aux généraux, aux armées, aux Collèges et aux magistrats ; ces ordres seront signés, selon l'importance de l'affaire, soit par S. M. Tsarienne et le grand chancelier, soit par le grand chancelier seul », écrivait Fick ${ }^{37}$.

En d'autres termes, Fick proposait de diviser le Sénat en départements fonctionnels communiquant avec le tsar grâce à la médiation du collège de la Chancellerie dirigé par le chancelier. À cette époque, la médiation entre Pierre Ier d'un côté et les institutions et les particuliers d'un autre côté était efficacement assurée par son Cabinet, composé d'un secrétaire, Aleksej Makarov, et quelques scribes ${ }^{38}$. On peut

32. S. M. Solov'ev, op. cit., vol. 8, p. 371.

33. Sekretnyj kollegium, Inostrannyh del kollegium, Posol’skaja kollegija. Voir S. A. Belokurov et al., eds, Očerk istorii Ministerstva inostrannyh del, 1802-1902 (Abrégé d'histoire du ministère des Affaires étrangères, 1802-1902), Saint-Pétersbourg, Ministerstvo Inostrannyh del, 1902, p. 36 ; S. M. Solov'ev, op. cit., vol. 8, p. 397, 415.

34. C. Peterson, op. cit., p. 62-67. Voir une biographie de Fick par Arno Cederberg, Heinrich Fick. Ein Beitrag zur russischen Geschichte des XVIII. Jahrhunderts, Tartu-Dorpat, Mattiesen, 1930.

35. Sur cette réforme, voir une bibliographie dans l'ouvrage de M. V. Babič et le livre de E. V. Anisimov, cités supra.

36. $Z A, \mathrm{n}^{\circ} 331$, p. 272-275.

37. Ibid., p. 272. Ici et plus loin, les citations en français sont traduites du russe par nous.

38. Sur le rôle de Makarov dans la chaîne de gouvernement voir E. V. Anisimov, op. cit., p. 282-290. Voir aussi S. M. Solov'ev, op. cit., p. 310 ; N. P. Eroškin, Istorija gosudarstvennyh učreždenij dorevoljucionnoj Rossii (Histoire des institutions de l'État en Russie prérévolutionnaire), 4 éd., Moscou, Tretij Rim, 1997, p. 70 ; Gosudarstvennost' Rossii, konec XV veka fevral' 1917 g. Slovar'-spravočnik (Les institutions politiques et administratives de Russie, fin XV - février 1917. Dictionnaire encyclopédique), Moscou, Nauka, 1999, vol. 2, p. 133$142 ; Z A$, n $^{\circ} 222$, p. 170-173, nº 227, p. 177-178. 
supposer que Fick, un Occidental s'inspirant du modèle gouvernemental suédois ${ }^{39}$, voyait en Makarov une espèce de secrétaire privé alors que le chancelier était pour lui un grand officier de la couronne. En dernière analyse, son projet consistait à établir une chaîne institutionnelle ininterrompue entre le tsar et les rouages exécutifs.

L'idée de Fick ne pouvait cependant pas être perçue par les lecteurs de son projet qui ignoraient presque tout des modèles qu'il gardait à l'esprit. Pierre I ${ }^{\mathrm{er}}$, pourtant très intéressé, ne prêta aucune attention à la spécificité du collège de la Chancellerie. En traduisant ce nom en russe, il le transforma en un collège de la Chancellerie des Affaires étrangères ${ }^{40}$. Cela signifiait que le tsar réduisit sa sphère de compétence à la seule direction du service diplomatique.

Ainsi, au lieu d'élever le chancelier et son département à la place d'intermédiaire entre le tsar et les institutions, le projet de Fick produisit un effet contraire. Le remplacement de l'appellation conseil secret par collège des Affaires étrangères, qui à été évoqué plus haut, exprimait une certaine dégradation de statut. Désormais, Golovkin, Šafirov, Dolgorukij et Tolstoj composaient non plus un conseil diplomatique auprès du souverain, mais la direction d'une structure administrative : ils formaient le collège qui gérait la chancellerie des Ambassades. Quant au Cabinet, Pierre Ier n'envisageait apparemment pas sa réforme.

Au reste, le projet d'établissement des collèges parut attrayant et son auteur digne de confiance. À la fin de 1715, Pierre Ier envoya Fick à Stockholm avec une mission secrète : rassembler des informations détaillées sur le système administratif suédois. Fick réussit très bien sa mission et quitta la Suède en décembre 1716, après douze mois de séjour. En janvier 1717, Fick vint voir le tsar à Amsterdam et lui fit le rapport de ses succès. Très satisfait, Pierre Ier prolongea son contrat de service et le désigna comme un des responsables de la réforme collégiale qui allait commencer ${ }^{41}$.

Le tsar rentra en Russie en octobre 1717. Grâce aux renseignements de Fick, il avait maintenant des informations plus précises sur le système collégial suédois et il composa une nouvelle liste des collèges à instituer. On n'y voyait plus le collège de la Chancellerie des Affaires étrangères, mais un collège Politique (Političeskaja kollegija). La description des fonctions de ce dernier (délivrer « tous les oukases du tsar et les lettres patentes et les ordres de mission $»^{42}$ ) correspond pourtant exactement à la description du collège de la Chancellerie donnée par Fick en 1715. Il s'agissait bien de la même chose. Le changement de nom montre cependant qu'entre-temps Fick s'était rattrapé : il avait informé le tsar du véritable rôle du collège de la Chancellerie de Stockholm.

39. Claes Peterson a démontré qu'en 1715 Fick pensait déjà au système collégial suédois (C. Peterson, op. cit., p. 62-67). Une comparaison entre ce projet et un autre mémoire de Fick sur le même sujet, écrit le 25 avril 1718 (ZA, no 414, p. 542-549), met cependant en évidence qu'en 1715 Fick n'avait pas encore cette connaissance intime, précise, des institutions suédoises, qu'il acquit plus tard.

40. Kanceljarii čužestrannyh del kollegium. Voir une note écrite de la main du tsar et datée de 23 mars 1715 (ZA, no 254, p. 213).

41. Ibid., no 266, p. 221.

42. Ibid., no 263 , p. 218. 
Pendant un moment, Pierre I ${ }^{\mathrm{er}}$ réfléchit sans doute à l'utilité d'une institution telle que le collège Politique. Sa décision fut négative. Le 15 décembre 1717, il désigna les présidents et les vice-présidents des collèges : le chancelier Golovkin et le vice-chancelier Šafirov furent nommés, respectivement, président et vice-président d'un collège des Affaires étrangères. Ni le collège de la Chancellerie ni le collège Politique ne furent mentionnés ${ }^{43}$. En même temps que l'institution des collèges, se déroula une réforme du Sénat qui devint une assemblée des présidents de collèges ${ }^{44}$.

Le Sénat et les collèges, tout semblait être fait comme Fick l'avait proposé en 1715. Mais, ayant acquis entre temps une connaissance plus profonde du système collégial suédois, il voyait que, dans la copie russe, la pièce maîtresse de l'original manquait : le collège de la Chancellerie qu'il se représentait comme un lien indispensable entre le monarque et le gouvernement, entre le souverain et son peuple. Toutefois, à ce stade de la réforme, Fick ne perdit pas encore l'espoir de la ramener dans la bonne voie. Le 25 avril 1718, il soumit au chancelier une Kurze Beschreibung der Königlichen Schvedischen Reichs-Kanzeley oder des Kanzeley-Kollegii in Stocholm, mémoire beaucoup plus long et circonstancié que son titre ne permet de le supposer ${ }^{45}$. Le but de Fick était de persuader Pierre Irr qu'une institution analogue au collège de la Chancellerie de Stockholm était indispensable. Pour commencer, il donnait une description détaillée du Kanslikollegium. En voici un résumé.

Après 1680, quand le roi de Suède redevint monarque absolu, et jusqu'en 1714, le collège de la Chancellerie n'était rien d'autre que le Cabinet du roi. Il était dirigé par deux ou trois ministres d'État, dont un, le chancelier, remplissait la fonction de président. Les ministres étaient assistés par des conseillers de la chancellerie et des secrétaires d'État qu'on appelait aussi secrétaires du roi. Le collège de la Chancellerie était chargé de toutes les affaires dont la décision dépendait directement du roi $^{46}$.

Les affaires étrangères et autres affaires secrètes n'étaient jamais livrées à la totalité du collège de la Chancellerie. Le roi en discutait avec un ou deux ministres d'État les plus proches ; un des conseillers de la chancellerie les assistait parfois. Les affaires intérieures, par contre, étaient examinées par tous les membres ensemble. Elles étaient distribuées entre les conseillers de la chancellerie qui étaient responsables de leur exécution.

43. Ibid, $\mathrm{n}^{\circ} 265$, p. 219-220.

44. E. V. Anisimov, op. cit., p. 240-242.

45. $Z A, n^{\circ} 414$, p. 542-549 (le texte est publié en russe, dans une traduction contemporaine de l'original).

46. Les négociations avec les États étrangers; les instructions et les ordres aux représentants diplomatiques du roi. Tous les ordres, notifications et explications du roi aux hauts magistrats et parfois même aux généraux. Les résolutions du roi sur les requêtes des particuliers. Les appointements des hauts dignitaires. La délivrance des diplômes et des lettres patentes pour les titres nobiliaires, les pensions, les terres. Tous les manifestes, les règlements et les confirmations qui devaient être publiés au nom du roi à l'intérieur ou à l'extérieur des frontières suédoises. La gestion des Postes. Les archives de l'État. La censure. 
Le collège de la Chancellerie ne pouvait rien ordonner ni aux autres collèges, ni aux gouverneurs des provinces ou autres magistrats. Ce n'étaient pas les membres du collège de la Chancellerie mais le roi lui-même qui signait tout ce qui en sortait ${ }^{47}$.

Les conseillers de la chancellerie et les secrétaires du roi étaient choisis parmi des serviteurs éprouvés, administrateurs expérimentés et gens de haute culture. Leurs appointements n'étaient pas plus élevés que dans les autres collèges. En revanche, ils faisaient l'objet d'un si grand respect que le roi leur confiait toutes les missions à l'étranger. Plusieurs conseillers et secrétaires du collège de la Chancellerie avaient directement été promus sénateurs et étaient devenus de grands ministres.

Une institution comme le collège de la Chancellerie était utile à un monarque absolu parce que, même si la législation de son pays était élaborée et les institutions administratives et judiciaires s'avéraient efficaces, comme en Suède, des questions surgissaient quotidiennement qui ne pouvaient être résolues sans l'intervention directe du souverain. Or, un roi n'avait pas toujours le temps de rentrer dans les détails de chaque affaire. Pourtant, les jugements et les décisions émanant de lui devaient être infaillibles. C'est là-dessus que veillait le collège de la Chancellerie. Il était donc un véritable gouvernail de toutes les affaires d'État et le gardien du prestige de la monarchie. En outre, quand le roi était absent du pays, le Sénat (c'est-àdire le Riksråd) et le collège de la Chancellerie le dirigeaient à sa place.

Il faut reconnaître que Fick donnait une présentation assez exacte du Kanslikollegium de Stockholm ${ }^{48}$. Une autre partie de son mémoire était consacrée à un éloge passionné de Charles XI.

Ce roi, expliquait Fick, n'avait pas reçu une éducation très soignée. Mais il avait eu un goût pour les savoirs, avait été naturellement intelligent et grand travailleur. Il avait tenu toutes les affaires de son État dans ses mains et contrôlé personnellement le fonctionnement des institutions. Avant le retour de la Suède dans la famille des monarchies absolues, le pays avait été plongé dans le désordre. Charles XI réforma tout et mena tout - lois et institutions - à un état de perfection suprême. Enfin, le roi laissa un testament en bonne et due forme où il expliquait comment gouverner l'État pendant la minorité de son fils ${ }^{49}$.

Force est de constater que Fick cherchait sciemment à attiser en Pierre Irr l'esprit d'émulation et à lui montrer quelles devaient être les grandes lignes de sa réforme. La fin du mémoire était consacrée à un objet qui intéressait spécialement son auteur.

Le Sénat et le collège de la Chancellerie, écrivait Fick, avaient limité le pouvoir royal. Mais Charles XI sauva les intérêts de la monarchie absolue. Il transforma le

47. Rappelons que, dans le mémoire de 1715, Fick écrivait, au contraire, que « les hauts ordres aux amiraux, aux généraux, aux armées, aux collèges et aux magistrats [...] seront signés, d'après l'importance de l'affaire, soit par S. M. et par le grand chancelier soit par le chancelier seul » $\left(Z A, \mathrm{n}^{\circ} 331\right.$, p. 272).

48. C. Peterson, op. cit., p. 381-384.

49. L'allusion est saisissante par sa hardiesse : le fils unique de Pierre Ier venait d'être destitué et son petit-fils n'avait que trois ans, mais le tsar hésitait toujours à faire un testament. 
Sénat en une Haute cour d'appel, et cette assemblée aristocratique perdit l'énorme pouvoir dans le gouvernement dont elle jouissait depuis des siècles. Quant au collège de la Chancellerie, le roi en fit son Cabinet. Il y nomma plusieurs ministres - qu'il choisissait, soulignait Fick, selon le mérite, non selon la naissance (il anoblissait les plus valeureux) - et décidait de toutes les affaires avec eux.

En évoquant le règne de Charles XII, Fick développa encore plus le motif de la coopération fructueuse entre le roi et ses conseillers intimes. Depuis 1714, ce roi n'usait plus des services du collège de la Chancellerie. Il choisit de gouverner par l'intermédiaire de six ministres d'État dont chacun avait son propre petit bureau composé d'un secrétaire d'État et de quelques commis (système d'inspiration française, remarquait Fick).

Le premier de ces six ministres, grand ombudsman, plénipotentiaire du roi dans les affaires de justice, séjournait à Stockholm, au Sénat. Les cinq autres portaient les titres d'ombudsrad, ou conseiller secret. Ils suivaient le roi toujours et partout. Le premier était responsable de l'armée et de la marine, le deuxième du trésor et des mines, le troisième du commerce et des manufactures, le quatrième des affaires étrangères hormis celles d'Allemagne, et le dernier des relations avec les cours germaniques. Aucun d'eux n'avait le droit de s'immiscer dans les responsabilités des autres. Il leur était également interdit de perturber le fonctionnement normal des collèges correspondants. Les ministres ne faisaient que les contrôler et servir d'intermédiaires entre le roi et les collèges, les magistrats et les généraux.

Ainsi, bien que le roi fût absent de la capitale des années durant, il n'avait plus besoin de Sénat pour le remplacer ; il ne tenait plus de séances au Sénat et ne lui demandait plus son conseil. Fick alla jusqu'à supposer que Charles XII avait voulu abolir tout à fait le titre de sénateur, parce que, dans ses conseils, il n'invitait plus personne en tant que sénateur et n'octroyait ce titre à personne.

La Kurze Beschreibung montre bien que l'idéal de Fick était le wohlgeordnete Polizeistaat. D'après Fick, le seul régime convenable pour un pays en voie de réformes globales était la monarchie absolue. Tout élément de l'aristocratie l'affaiblissait. Par conséquent, la compétence du Sénat - que Fick interprétait comme une institution aristocratique par définition - devait être limitée au domaine judiciaire. D'autre part, le monarque absolu ne pouvait échapper à sa nature humaine et avait besoin d'assistants : conseillers intimes, hommes hautement éclairés, administrateurs expérimentés, élevés à cette suprême confiance grâce à leurs mérites et non pas à leur naissance.

Sur ce dernier point, le discours de Fick n'était pas totalement désintéressé. Ce n'était pas un hasard s'il terminait son mémoire par l'histoire «d'un étranger » que Charles XII s'était attaché pour « lui confier temporairement la direction suprême des revenus, grâce à quoi ses finances furent remises en bon état $»^{50}$. Fick ambition-

50. ZA, no 414, p. 549. Il s'agit de Georg Heinrich von Görtz (1668-1719). D’origine hessoise, ministre des Finances de Charles XII, il fut condamné à mort après la disparition du roi, pour haute trahison. 


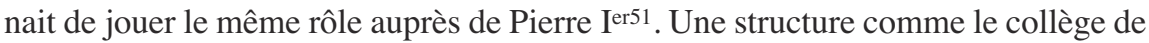
la Chancellerie lui aurait offert un cadre propice pour réussir ce genre de carrière.

Or, la réforme collégiale en Russie ne se déroulait pas exactement comme Fick l'avait prévu. Au lieu de fonder un collège de la Chancellerie, Pierre Irer continuait à gouverner par l'intermédiaire de deux structures également insatisfaisantes aux yeux de Fick : le Cabinet personnifié par Makarov, simple commis qui ne pouvait aucunement prétendre à la caractéristique d'un homme de haute culture, et le Sénat majoritairement aristocratique (en 1718, parmi les quatorze sénateurs, il y avait deux généraux étrangers, deux roturiers favoris du tsar et dix descendants de familles aristocratiques russes ${ }^{52}$ ).

Fick ne pouvait pas être sûr que la Kurze Beschreibung fût parvenue jusqu'au tsar $^{53}$ et sentait en tout cas que Pierre Ir avait une idée trop vague des institutions suédoises ${ }^{54}$. Toutefois, Fick avait encore une occasion formidable pour s'exprimer et être écouté, sinon entendu : très vraisemblablement, on lui avait confié la rédaction d'un projet de Règlement général des collèges russes (General'nyj reglament $)^{55}$.

En décembre 1718, Pierre I ${ }^{\text {er }}$ reçut la traduction russe de son projet intitulée Le très gracieux Règlement général de $S$. M. Tsarienne ${ }^{56}$. Le document commençait par une liste de neuf collèges. Tous les noms, sauf un, correspondaient aux noms des collèges institués par Pierre Ier en décembre 1717 : à la place du collège des Affaires étrangères on voyait, bien sûr, le collège de la Chancellerie.

La substitution trouvait son explication dans l'article III du projet ${ }^{57}$. Le collège de la Chancellerie devrait servir d'intermédiaire entre le tsar (ou, pendant ses absences, le Sénat) et tous les autres collèges. Ce collège comprendrait plusieurs ministres, chargés de préparer des rapports pour le tsar et expédier ses ordres. Chaque ministre serait responsable d'un domaine spécifique des affaires d'État.

51. En 1717, le tsar l'avait nommé conseiller du futur collège des Revenus (C. Peterson, op. cit., p. 75). Quand ce collège commença à fonctionner, Fick se lassa rapidement du travail routinier mené par ses membres. Il ne voulait plus siéger au conseil collégial : « weilen ich wegen Mangel der Sprache und andern Ursachen, die beste Zeit und Morgen-Stunden im CammerCollegio meistentheils unnütz passiren muss, in welcher ich doch zu Hause wichtigere Dienste leisten, und daneben doch, so viel es von mir dependieret, dass Cammerwesen in gewissen Tagen oder Stunden befordern könte, so wird es Ihro Majtt: Gnädigstem Gutfinden in tieffester Unterthänigkeit übertragen, wie sie über meiner geringen Persohn und künftigen Verrichtungen zu disponiren Gnädigst geruhen wollen », écrivait-il à Pierre I ${ }^{\mathrm{er}}$ (A. Cederberg, op. cit., Beilage 4, p. 104). Fick aurait préféré travailler directement avec le tsar, devenir son conseiller personnel en matière financière et économique.

52. D'après une liste alphabétique de sénateurs publiée dans Istorija Pravitel'stvujuščego Senata za 200 let, 1711-1911 (Histoire du Sénat Gouvernant, 1711-1911), Saint-Pétersbourg, 1911, vol. 5, p. 109-161.

53. ZA, no 332, p. 277.

54. C. Peterson, op. cit., p. 92. Dans un autre mémoire, du 9 mai 1718, Fick priait le tsar de se faire lire des descriptions des collèges suédois $\left(Z A, \mathrm{n}^{\circ} 269\right.$, p. 224).

55. C. Peterson, op. cit., p. 117-119.

56. Ego Carskogo Veličestva milostivejšij General'nyj reglament : ZA, no 400, p. 413-453.

57. Ibid., p. 417-418. 
Chaque ministre aurait son propre assistant : un secrétaire d'État, ou conseiller de la chancellerie. Ce dernier pourrait remplacer son ministre en cas d'absence. Il faudrait veiller à ce que les ministres russes aient des assistants étrangers et vice versa (une allusion à Fick lui-même).

En 1715, Fick avait décrit le collège de la Chancellerie principalement comme un rouage de gestion des affaires diplomatiques. Dans la Kurze Beschreibung, il se corrigea en montrant que les affaires intérieures occupaient le collège de la Chancellerie autant, sinon plus, que les affaires extérieures. Maintenant, il poussait encore plus loin : la diplomatie, comme quelque chose allant de soi, n'était même pas spécialement mentionnée, l'essentiel était de souligner que le collège de la Chancellerie portait la responsabilité de toutes les affaires dont la décision dépendait personnellement du tsar.

L'article IV du projet ${ }^{58}$ traitait du Sénat. Fick soulignait l'importance secondaire du Sénat en tant qu'institution temporaire. Le Sénat ne devait pas avoir d'existence propre, il était un mandataire que le tsar laissait sur place quand il partait de la capitale. Le collège de la Chancellerie préparerait des rapports et expédierait les décisions du Sénat exactement de la même façon que pour le tsar, à une exception près : devant le Sénat, les rapports seraient faits non par les ministres eux-mêmes mais par les secrétaires d'État (conseillers de la chancellerie).

Il est évident que les articles III et IV résumaient la Kurze Beschreibung ${ }^{59}$. Fick pencha finalement pour le modèle du Cabinet de Charles XII et non pour celui du collège de la Chancellerie de Charles XI : les ministres n'étaient pas censés tenir des séances communes. Pour une raison ou une autre Fick ne voulait pas, dans ce cas, promouvoir le principe de la collégialité, mais ne pouvait pas l'abandonner ouvertement : il savait que ce principe était devenu pour Pierre Ier le cœur même de toute la réforme. Fick maintint donc, un peu naïvement, le nom de collège de la Chancellerie, où le mot collège ne figurait plus que pour la pure forme.

Pierre Ier ordonna de distribuer des exemplaires du Très gracieux Règlement général aux présidents des collèges, qui devaient donner leurs avis sur ces points ${ }^{60}$. L'article III attira immédiatement l'attention'61. Fin décembre 1718 (début janvier 1719), Pierre Ir donna l'ordre de remplacer les articles III et IV du Règlement général par un document de sa propre rédaction, les Fonctions du Sénat (Dolžnost' Senata ${ }^{62}$. Cela signifiait que le tsar rejetait une nouvelle fois les propositions de Fick concernant la création du collège de la Chancellerie et la révision du statut et des fonctions du Sénat. Quels pouvaient être les motifs de sa décision?

Premièrement, Pierre I ${ }^{\mathrm{er}}$ voyait sans aucun doute très clairement que la plupart des fonctions du Sénat correspondaient à celles du collège de la Chancellerie de

58. Ibid., p. 418.

59. C. Peterson, op. cit., p. 388-389.

60. $Z A$, n $^{\circ} 411$, p. 453.

61. Dans l'exemplaire qui se trouvait au Cabinet de Pierre Ire on peut voir une note anonyme, en marge de cet article : « faire un rapport à S. M. » (ibid., n 400, p. 417).

62. Comparez les versions B, V, G et D du Règlement général (ibid., p. 417-418, 454, 458). 
Fick $^{63}$ (il faut rappeler que, à ce moment précis, Pierre I ${ }^{e r}$ travaillait sur le texte des Fonctions du Sénat $\left.{ }^{64}\right)$. Contrairement au collège de la Chancellerie, le Sénat ne s'occupait pas de la diplomatie, mais ce domaine avait été confié au collège des Affaires étrangères (de même que la gestion des Postes et des Archives). Enfin, le rôle central de médiation entre le tsar et les institutions, dont Fick aurait voulu doter le collège de la Chancellerie, était joué par Makarov, dont Pierre I ${ }^{\mathrm{er}}$ paraissait toujours être satisfait. En un mot, le Sénat, le collège des Affaires étrangères et le Cabinet remplissaient déjà toutes les fonctions dont Fick voulait donner la charge au collège de la Chancellerie, et Pierre ${ }^{\mathrm{er}}$ ne voyait certainement pas l'intérêt de combiner ces trois institutions en une seule 65 .

Deuxièmement, Pierre $\mathrm{I}^{\mathrm{er}}$ ne semblait pas craindre que des clans aristocratiques pussent entraver son pouvoir personnel par leur simple représentation au Sénat.

Et troisièmement, d'après sa propre affirmation, Pierre Ier était toujours prêt à écouter les conseils de ses sujets et à remplir ainsi l'obligation morale incombant aux monarques absolus cherchant le bien public ${ }^{66}$. Il ne lui était pas nécessaire, pour cela, de s'entourer d'un cercle choisi et restreint de spécialistes du conseil. Ses sujets le savaient qui lui adressaient toutes sortes de projets de réformes petites et grandes ${ }^{67}$.

Cette fois, Fick comprit qu'il était inutile d'insister plus longtemps et ne plaida plus la cause du collège de la Chancellerie. Son espoir de faire une carrière dans le cadre de cette institution chimérique s'évapora. Désormais, il adopta pour s'élever une stratégie moins sophistiquée, plus commune et, si l'on peut dire, plus égoïste, qui consistait à frayer son chemin tout seul : en 1724, on vit Fick prier Pierre Ier de le nommer son conseiller personnel pour les affaires économiques ${ }^{68}$.

63. Le Sénat publiait les oukases, faisait exécuter les ordres du tsar et contrôlait leur exécution. Il était chargé de l'examen des requêtes (sur la procédure de réception et d'examen des requêtes, voir ibid., nos 371 à 395). Il devait résoudre les questions qui dépassaient les pouvoirs ou les compétences des collèges et des gouverneurs. Il devait élire de hauts magistrats, dont les membres des collèges. Il effectuait les promotions en grade. Il était le dépositaire des sceaux de l'État qu'on apposait sur les lettres patentes, les diplômes, etc. (oukase du 10 décembre 1718 : ibid., $\mathrm{n}^{\circ}$ 58, p. 64-65). Enfin, le Sénat de Pierre Irr avait un ample pouvoir consultatif : quand il fallait introduire de nouvelles lois ou modifier des anciennes, il devait préparer des projets ou des amendements et en faire un rapport au tsar qui prenait ensuite sa décision. L'oukase définissant le pouvoir consultatif du Sénat date du 17 avril 1722 (ibid., no 132, p. 107-108), mais en pratique le Sénat participa au travail législatif dès le début de son existence.

64. Ibid., p. 281.

65. E. V. Anisimov, op. cit., p. 121.

66. Propos de Pierre Ier rapportés par A. K. Nartov: L. Majkov, Rasskazy Nartova o Petre Velikom (Les anecdotes de Nartov à propos de Pierre le Grand), Saint-Pétersbourg, 1891, p. 82 .

67. N. P. Pavlov-Sil'vanskij, Proekty reform v zapiskah sovremennikov Petra Velikogo: Opyt izučenija russkih proektov i neizdannye ih teksty (Projets de réformes dans les mémoires des contemporains de Pierre le Grand : Essai d'une étude des projets russes et leurs textes inédits), nouvelle éd., Moscou, Gosudarstvennaja publičnaja istoričeskaja biblioteka Rossii, 2000.

68. C. Peterson, op. cit., p. 412. Rien n'indique que Pierre I ${ }^{\mathrm{er}}$ ait satisfait cette demande. Toutefois, en 1726, Catherine Ire accorda à Fick le grade de conseiller d'État et proposa de le nommer vice-président du collège du Commerce (SIRIO, vol. 55, p. 428), ce qui fut fait (C. Peterson, op. cit., p. 76). 


\section{La réception des projets de Fick : Andrej Osterman}

Cependant, Fick ne prêchait pas dans le désert. On l'écoutait, on l'entendait. Andrej Osterman (futur membre du Conseil suprême secret et vice-chancelier) fut l'un des hommes qui essayèrent de reprendre à leur compte et de promouvoir certaines des idées de Fick.

Osterman et Fick, dont les relations personnelles ne sont pas connues, avaient beaucoup en commun. Allemands, protestants, ils appartenaient à une même génération et avaient fait des études universitaires. De condition non noble, ils avaient dû commencer leur service à un niveau assez bas, tous deux à l'étranger. Ambitieux et travailleurs, ils avançaient relativement vite et cherchaient un emploi susceptible de leur offrir des activités indépendantes, des responsabilités personnelles ${ }^{69}$.

Osterman avait toutefois des avantages sur Fick. Il était arrivé en Russie onze ans plus tôt, il avait appris le russe et s'était parfaitement acclimaté dans le nouveau pays. Dès le début de son service en Russie, Osterman s'était trouvé dans l'entourage immédiat du tsar ${ }^{70}$. Il n'avait bien sûr occupé qu'une place très modeste dans les rangs des commis et des traducteurs, mais cela lui avait permis de faire valoir ses mérites, de se faire remarquer, de devenir nécessaire. L'ambition d'Osterman était encouragée par l'exemple de la vertigineuse carrière de son collègue Šafirov, qui, sans avoir fait d'études universitaires, avait réussi à s'élever d'un milieu social plus bas, grâce uniquement à ses talents naturels et à l'habileté à trouver un bon patron, ou plutôt, le bon patron : le tsar.

Osterman n'était certainement pas moins talentueux que Šafirov, mais cette dernière capacité, indispensable, lui faisait défaut : il ne sut jamais gagner la sympathie de Pierre Irr. Par conséquent, la carrière d'Osterman, très brillante, était en même temps beaucoup plus progressive que celle de Šafirov. Osterman parvenu au grade de conseiller secret et au titre de baron (1721), Pierre Ier hésitait à le promouvoir davantage ${ }^{71}$. Šafirov disgracié en 1722, Osterman était le seul candidat à sa succession. Néanmoins, Pierre Ir ne voulut pas lui octroyer le grade de vicechancelier, bien qu'il remplît les fonctions de vice-président du collège des Affaires étrangères.

69. M. A. Polievktov, «Andrej Ivanovič Osterman», in Russkij biografičeskij slovar' (Dictionnaire biographique russe), Saint-Pétersbourg, 1905, vol. 12, p. 405 ; C. Peterson, op. cit., p. 72-73.

70. S. M. Solov'ev, op. cit., p. 310.

71. Détail important, même ce grade et ce titre, Osterman les avait reçus dans des circonstances assez particulières. En signant la lettre patente, Pierre I ${ }^{\mathrm{er}}$ avait inscrit, en bas, la phrase suivante : «Déclarer cela [la nomination et l'octroi du titre] lors de la signature du traité pour ne pas faire reculer la frontière près de Rogsli » (RGADA, f. 142, op. 2, d. 11, 1. 263). Le tsar sousentendait le traité de paix de Nystad, Osterman étant l'un des deux représentants du tsar au congrès. Il ne s'agissait donc pas d'une récompense ni, encore moins, d'une marque de faveur personnelle. Ruse diplomatique courante, cette promotion était censée amplifier le prestige d'Osterman, le mettre à l'échelle des plénipotentiaires suédois et lui rendre les pourparlers plus faciles. 
Moins aimé que le favori Menšikov et même que Šafirov, dépourvu d'une généalogie imposante, Osterman devait continuer à rendre des services importants s'il voulait continuer à avancer. Pour avoir l'occasion de le faire, il fallait être bien placé. Osterman se trouvait donc devant le même problème que Fick : nouer avec le souverain une relation qui fût fondée non pas sur un sentiment personnel, mais sur un rapport institutionnel entre un employeur et un employé ; un rapport cependant aussi direct, privilégié et protégé que le sont les relations d'amitié, d'amour ou de parenté. Cela devait amener Osterman à réfléchir à une institution qui donnerait un cadre formel à la coopération entre le monarque et ses conseillers.

Osterman avait sous la main nombre de documents susceptibles de nourrir sa pensée sur le sujet. Les écrits de Fick concernant le collège de la Chancellerie devaient forcément l'intéresser et il y avait accès, puisque les projets étaient conservés au collège des Affaires étrangères. Par ailleurs, Osterman avait des connaissances directes des institutions suédoises, acquises lors de son séjour au congrès d'Åland en 1718-171972. En 1719, Osterman consulta également plusieurs mémoires concernant l'organisation des États européens, et notamment les mémoires de Matveev déjà mentionnés ${ }^{73}$, ainsi que des copies des règlements des rois de Danemark et de Norvège, Christian V (1699) et Frédéric IV (1717), qui le renseignèrent sur la composition des conseils privés des monarques danois ${ }^{74}$.

Osterman utilisa tous ces matériaux dans un projet de la Table des rangs qu'il rédigea, sur un ordre du tsar, à la fin de 1719 et au début de $1720^{75}$. Mais, par ailleurs, il entreprit de synthétiser ce qu'il avait appris sur les conseils et les cabinets des monarques européens. Parmi ses papiers se trouve un texte inachevé, intitulé Proposition composée d'après des règlements des rangs ${ }^{76}$. Ce texte - une simple ébauche, une série de notes et d'idées encore imprécises - présente toutefois un grand intérêt.

En premier lieu, l'auteur cherchait à donner une description d'une institution qu'il appelait tantôt cabinet, tantôt conseil secret :

Messieurs les sénateurs sont différents et ont des rangs différents parce qu'ils président des bureaux et des collèges. Les conseillers d'État actuels, ministres du Cabinet secret, ont à bon droit un rang plus élevé que tous les autres [sénateurs] et sont les plus importants. Parce qu'ils gardent sous secret, rapportent au souverain et expédient des affaires d'État intérieures et extérieures, comme les traités de paix, les promotions aux grades, les instructions aux ambassadeurs. Ils lisent les dépêches diplomatiques et en font des rapports, ils accueillent les ambassadeurs étrangers, tiennent des conférences avec eux et leur donnent

72. S.A. Fejgina, Alandskij kongress. Vnešnjaja politika Rossii v konce Severnoj vojny (Le congrès d'Åland. La politique extérieure russe à la fin de la guerre du Nord), Moscou, 1959, p. 409.

73. Osterman les étudia et en fit extraire des résumés. Voir S. M. Troickij, op. cit., p. 57-58.

74. Ibid., p. 61

75. Ibid., p. 48-64.

76. Predloženie iz inyh rang reglamentov sočinennoe (RGADA, f. 370, op. 1, d. 16, 1. 50-56). 
congé. S. M. transmet par leur intermédiaire ses oukases au Sénat, aux généraux et aux hauts magistrats ${ }^{77}$.

Ce passage ressemble de près à la description des fonctions du collège de la Chancellerie donnée par Fick dans ses mémoires de 1715 et de $1718^{78}$. Seulement, Osterman, voulant éviter tout malentendu, remplaça le mot collège, qui évoquait désormais un rouage administratif, par le mot cabinet. Soulignons que les membres du Cabinet, et d'abord le chancelier, font partie du Sénat mais se placent au-dessus des autres sénateurs :

À Rome et dans d'autres États, le grade de grand chancelier est le premier et le plus illustre ; en France, en Angleterre et en Danemark, le grand chancelier préside le Conseil secret ou le Parlement. Les amiraux, les feld-maréchaux et les généraux font partie de ce Cabinet et ont le même rang [que les conseillers d'État actuels].

Les conseillers d'État actuels ne se réunissent pas régulièrement dans un collège particulier, mais sont employés à des conférences et à des commissions. Ils sont aussi envoyés avec des missions secrètes extraordinaires ${ }^{79}$.

En second lieu, l'auteur s'appliquait à démontrer, sur des exemples historiques, que les conseils secrets étaient l'assise institutionnelle principale de la monarchie absolue. Les souverains, écrivait-il, ont communément un grand nombre de conseillers, ils suivent en cela l'enseignement de la Bible qui dit : « Là où se trouvent maints conseillers, on agit convenablement ${ }^{80}$. L'Espagne avait été un État très puissant. Mais ses rois n'avaient pas eu de conseils où les affaires auraient été partagées entre les conseillers selon la spécialisation de chacun. C'est pourquoi la grandeur de ce pays finit par tomber. Par contre, certains princes « de force moyenne » instituèrent des conseils en bonne et due forme où ils examinaient, entourés de sages, toutes les affaires. Ils parvinrent ainsi à établir un bon ordre dans les affaires intérieures et extérieures de leurs États ${ }^{81}$.

Osterman ne termina pas son mémoire. Occupé comme il l'était par le projet de la Table des rangs, puis envoyé au congrès de Nystad, il avait peu de temps à y consacrer. Il pensait peut-être aussi que le tsar n'accueillerait pas de bon cœur ses arguments. Pierre Ier n' avait pas de conseil officiel, mais il venait d'élever la Russie au comble de la gloire. Pouvait-il croire pertinente une comparaison entre son propre gouvernement et la défaillante machine étatique d'Espagne ? Osterman temporisait, il savait le faire. Entre-temps, ses collègues lisaient, eux aussi, les projets de Heinrich Fick.

77. Ibid., 1. 50 .

78. $Z A, \mathrm{n}^{\circ} 331$, p. 272 et no 414, p. 542.

79. RGADA, f. 370, op. 1, d. 16,1. 50-50 ob.

80. L'auteur sous-entend apparemment un vers du Livre des proverbes, XI, 14. L'origine protestante d'Osterman se fait clairement sentir à travers cette citation biblique.

81. RGADA, f. 370, op. 1, d. 16,1. 51 ob.-52 ob. 


\section{La réception des projets de Fick au collège des Affaires étrangères}

Les membres du collège des Affaires étrangères étudièrent attentivement la Kurze Beschreibung de Fick, dans le cadre de leur travail sur un projet de règlement de leur collège ${ }^{82}$. Le principal rédacteur de ce projet, Vasilij Stepanov, et son chef, le chancelier Golovkin (respectivement, futur secrétaire et futur membre du Conseil suprême secret ${ }^{83}$ ), étaient visiblement intéressés par l'idée maîtresse du mémoire de Fick, à savoir que les affaires étrangères devaient être placées sous le contrôle immédiat du souverain qui les dirigerait en consultant ses ministres, régulièrement encadrés dans une sorte de conseil ou de cabinet. Voici ce que Stepanov écrivait dans une version de son projet :

Dans des États étrangers, dont la Suède, on peut avoir trois, quatre ou cinq ministres pour donner des conseils sur ces affaires [les affaires étrangères] ; dans certains pays, ils portent le nom de conseillers secrets de cabinet, dans d'autres de conseillers secrets actuels, chez l'Empereur ils ont pour nom conseillers secrets de conférence, alors qu'en Suède on les appelle conseillers du roi et sénateurs des Affaires étrangères ${ }^{84}$.

Par analogie, Golovkin, Šafirov, Dolgorukij et Tolstoj qui dirigeaient les affaires étrangères en Russie, sont évoqués dans ce projet comme «ministres d'État du Conseil secret $»^{85}$. Stepanov proposait également d'établir un registre spécial pour inscrire les noms des personnes élevées aux «grades récemment introduits par S. M. Tsarienne qui appartiennent au Conseil secret : le chancelier, le vice-chancelier, les conseillers secrets actuels et les conseillers secrets ${ }^{86}$. Cependant, tous ces passages - et en général toute mention du conseil secret - disparurent de la version définitive du projet qui fut présentée au tsar ${ }^{87}$.

Tout cela signifie que les membres du collège des Affaires étrangères auraient préféré être subordonnés non point au Sénat, avec tous les autres collèges, mais directement au souverain. Ils auraient voulu faire partie d'un conseil intime, semblable aux conseils secrets ad hoc que Pierre Ier avait tenus en 1711, mais dûment institutionnalisé et permanent.

82. C. Peterson, op. cit., p. 389-391.

83. Stepanov, la main droite du chancelier, reste pratiquement ignoré des historiens. Un canevas de sa carrière a été reconstitué par M. V. Babič, Gosudarstvennye učreždenija XVIII veka: komissii petrovskogo vremeni (Les institutions d'État au XVIII siècle : les commissions de l'époque pétrovienne), Moscou, Rossijskaja političeskaja enciklopedija, 2003, p. 455.

84. $Z A$, no $^{\circ} 409$, p. 530.

85. Ibid., p. 531.

86. Ibid., p. 534. Dans la première et la deuxième version du projet l'expression conseil secret n'avait pas encore été utilisée (RGADA, f. 370, op. 1, d. 12,1. 15).

87. $Z A, \mathrm{n}^{\circ} 406$, p. 519. Dans le brouillon de la version définitive, les mots conseil secret sont encore présents ; il furent donc enlevés au dernier moment (RGADA, f. 370, op. 1, d. 12,1.90). 
Ces insinuations ne furent pas entendues. Le 13 février 1720, Pierre Irr confirma un acte qui réglementait le fonctionnement du collège des Affaires étrangères ${ }^{88}$. Il entérina le mode de direction des affaires extérieures devenu habituel depuis plusieurs années : le tsar prenait les décisions soit tout seul, soit après une consultation informelle avec des conseillers secrets actuels qu'il invitait occasionnellement, mais jamais de façon régulière, dans son cabinet ou dans les locaux du collège des Affaires étrangères.

Quelques années après cet échec, en 1724, Osterman reprit la plume pour rédiger un mémoire destiné à l'attention de Pierre Ier : Propositions sur la composition et l'organisation de la chancellerie du collège des Affaires étrangères ${ }^{89}$. L'expression conseil secret y figure de nouveau :

Les affaires du ressort du collège des Affaires étrangères, ou, pour dire simplement, $d u$ Conseil secret, sont des affaires de la plus grande importance ${ }^{90}$.

En développant son étonnante affirmation, le vice-chancelier intérimaire attirait l'attention du tsar sur un fait qui lui paraissait évident : le collège des Affaires étrangères n'était pas un collège comme les autres, il ne pouvait pas être inconditionnellement subordonné au Sénat et les problèmes qu'il traitait n'étaient pas « de nature à être gérés strictement d'après le Règlement général ${ }^{91}$. Ce que le tsar aurait pu penser sur ce projet resta inconnu : il mourut avant d'avoir lu les Propositions ${ }^{92}$.

De retour au point de départ chronologique de cet article, quelques conclusions préliminaires s'imposent. Faire partie d'un conseil intime institutionnalisé auprès du souverain... Il semble bien que ce projet hantait les esprits de certains membres du gouvernement de Pierre I ${ }^{\mathrm{er}}$ depuis le début du siècle et surtout depuis le lancement de la réforme collégiale. Il attirait les outsiders élevés par la stratégie méritocratique de Pierre Ir , tels que Fick et Osterman. Les dignitaires de haute naissance, comme Golovkin, Dolgorukij ou Tolstoj, y trouvaient aussi leur compte. Les uns et les autres voulaient aménager un espace protégé à proximité immédiate du souverain. Pour les premiers, c'était le seul moyen d'amortir le handicap de leur naissance roturière et/ou de leur origine étrangère, et de s'inscrire durablement dans l'élite gouvernante. Les seconds espéraient obtenir un statut de conseillers officiels du tsar, qui les distinguerait de tous les autres participants des consultations organisées par le monarque.

88. Opredelenie Kollegii inostrannyh del ( ZA, n 406, p. 520-521).

89. K sočineniju i opredeleniju kanceljarii Kollegii inostrannyh del predloženija. Arhiv vnešnej politiki Rossijskoj imperii (Archives de la politique extérieure de l'Empire de Russie, cité infra AVPRI), f. 2, op. 6, d. 1, 1. 158-164 ob. Partiellement publié dans Očerk istorii Ministerstva inostrannyh del, op. cit., p. 40-46.

90. Ibid., p. 44. C'est nous qui soulignons.

91. Cité d'après S. L. Turilova, Istorija vnešnepolitičeskogo vedomstva Rossii (17201832 gg.) (Histoire du département des Affaires étrangères de la Russie (1720-1832)), Moscou, Institut Psihologii Rossijskoj Akademii Nauk, 2000, p. 18.

92. Voir un témoignage des membres du collège des Affaires étrangères dans un rapport adressé au Sénat en 1725 (AVPRI,f. 2, op. 6, d. 7,1. 1 ob.). 
Pierre $\mathrm{I}^{\mathrm{er}}$ ne semblait cependant pas favoriser l'idée de la création d'un conseil secret et, dans la plupart des cas, cette idée demeurait écartée d'une discussion ouverte. Les mots conseil secret étaient sur le bout des langues, mais ne parvenaient pas à franchir la barrière qui sépare un jargon professionnel d'un langage officiel.

Toujours est-il qu'entre 1710 et 1725 , le concept de conseil secret institutionnalisé fut peu à peu apprivoisé. La disparition de Pierre I ${ }^{\mathrm{er}}$, le règne chancelant de Catherine Ire et la nécessité de créer un front d'opposition aux prétentions exorbitantes du favori en titre, Menšikov, qui mettaient en danger l'existence politique, voire physique, des autres membres du gouvernement, toutes ces circonstances réunies créèrent une situation idéale pour ressusciter le projet de conseil secret. Il convient maintenant d'analyser la façon dont il fut réalisé.

\section{La création du Conseil suprême secret}

Les préparatifs qui précédèrent l'instauration du Conseil suprême secret sont très mal documentés. Seuls peuvent être mentionnés quelques indices indirects. Il fallait, évidemment, que les intéressés obtinssent l'accord de l'impératrice. Comment persuader Catherine Ire qu'une institution dont son mari n'avait pas favorisé la création était maintenant indispensable ? Avant de prendre une décision, Pierre Irr s'enquérait souvent de l'expérience européenne. Sa femme consentirait sans doute à suivre son exemple. Courant 1725, Osterman demanda à l'envoyé russe à Berlin, le comte A. G. Golovkin (fils du chancelier), une description détaillée des grades de la cour de Prusse. Il la reçut le 13 septembre $1725^{93}$. Puis, le 2 décembre 1725, le chancelier se fit apporter des archives du collège des Affaires étrangères Une brève description des grades de la Cour de France de Matveev dont il envoya le jour même une copie à Menšikov et à d'autres membres du collège de la Guerre $^{94}$. Le subit regain d'intérêt pour ce genre de documentation ne s'explique pas, comme on aurait pu le penser, par une intention de modifier la Table des rangs ${ }^{95}$. Le but était plutôt de persuader la nouvelle impératrice que l'établissement d'un conseil ou d'un cabinet secret ne présentait aucun risque pour son pouvoir personnel, puisque tous les monarques d'Europe en avaient un ${ }^{96}$. L'accord fut apparemment atteint lors d'un des rassemblements informels des conseillers secrets actuels de 1725 évoqués au début de l'article.

93. S. M. Troickij, op. cit., p. 121.

94. Ibid., p. 121-122.

95. Ibid., p. 122.

96. Le même scénario se répéta en 1730 , après la destruction des fameuses Conditions. Le 8 avril 1730, quelques semaines après l'avènement d'Anna Ivanovna, un secrétaire du collège des Affaires étrangères et confident de l'impératrice, Brevern, fit chercher aux archives des copies de tous les mémoires sur les grades des pays d'Europe ; au même moment, des rumeurs sur l'établissement d'un cabinet ou d'un nouveau conseil commencèrent à circuler (S. M. Solov'ev, op. cit., vol. 10, p. 262). Cette documentation fut étudiée avec attention, mais la Table des rangs ne subit aucun changement (S. M. Troickij, op. cit., p. 122). En revanche, Anna Ivanovna ordonna la création d'un Cabinet des ministres. 
Le Conseil commença à fonctionner le 9 février 1726 et ses premières résolutions portaient sur sa propre réglementation ${ }^{97}$. Durant le mois de février et jusqu'à la fin du mois de mars 1726, les membres du Conseil revinrent plusieurs fois sur cette question ${ }^{98}$. De ce point de vue, ils agissaient en parfait accord avec les principes de normalisation institutionnelle - au sens d'élaboration de règles explicites et officielles - chers au défunt tsar ${ }^{99}$.

Les procès-verbaux du Conseil révèlent les idées de ses membres sur les propriétés de la nouvelle institution. Les dispositions réglementaires du Conseil prises au jour le jour furent rassemblées dans un document déjà évoqué contenant treize articles, l'Avis sur le nouveau Conseil secret qui est institué. Cet Avis fut présenté à la séance du 23 février 1726 par le duc Karl-Friedrich von HolsteinGotthorp qui venait d'être nommé membre du Conseil ${ }^{100}$. Le texte fut lu, discuté et corrigé article par article. Menšikov et Osterman étaient absents de cette séance. Mais deux jours plus tard Menšikov emprunta un exemplaire de l'Avis pour prendre connaissance de son contenu ${ }^{101}$, et Osterman présenta peu après ses remarques qui furent lues devant le Conseil le 2 mars $1726^{102}$. Le 11 mars, le Conseil fit transmettre une version révisée de l'Avis à l'impératrice qui la rendit, avec ses corrections et ses remarques, le 16 mars 1726103. Modifié en conséquence, l'Avis fut finalement signé par les membres du Conseil le 21 mars $1726^{104}$.

La question de l'auteur du manuscrit initial n'est pas des plus simples. Le duc de Holstein prétendait l'être ${ }^{105}$. Mais il ne savait pas le russe ${ }^{106}$. Or, Vasilij Stepanov, qui dressait les procès-verbaux des séances du Conseil et se montrait généralement attentif à ce genre de détails, n'écrivit nulle part que l'Avis avait été initialement rédigé en allemand. Ou bien Stepanov omit de mentionner que le document avait été traduit, ou bien le duc n'était qu'un prête-nom, et dans ce cas le problème de l'auteur principal, celui qui préféra rester dans les coulisses, reste ouvert ${ }^{107}$.

97. Procès-verbal du Conseil suprême secret du 9 février 1726 (SIRIO, vol. 55, p. 2).

98. Ibid., p. 1-3, 25, 54-59, 63-65, 68-69, 90, 92-97, 98, 120, 124 (procès-verbaux).

99. Remarque importante de A. B. Kamenskij, op. cit., p. 193.

100. SIRIO, vol. 55, p. 57. Catherine Ire avait introduit le duc, son gendre, dans le Conseil, le 17 février 1726 (ibid., p. 43). Le duc était le mari de la fille aînée de Pierre Irer, Anna. En tant que membre de la famille impériale, il occupa la première place parmi les membres du Conseil (ibid., p. 56, 58, 65).

101. Ibid., p. 63-64.

102. Ibid., p. 68.

103. Ibid., p. 92, 96-97.

104. Ibid., p. 124.

105. Ibid., p. 57.

106. Ibid., p. 49, 98, 211.

107. Il n'est pas impossible que Fick fût le véritable rédacteur de l'Avis. Il avait jadis été au service du duc de Holstein, qui le connaissait personnellement depuis 1710 (C. Peterson, op. cit., p. 72-73). 
En tout cas, l'Avis fut un ouvrage collectif. Il est très important de souligner que tous les membres du Conseil, y compris l'impératrice, participèrent à plusieurs reprises, de façon plus ou moins active, à son élaboration. En outre, la clarté des énoncés de ce document indique que nous avons affaire à un texte dont les idées avaient longuement mûri, qu'elles étaient présentes, diffuses dans la pensée politique russe de l'époque et que les mots destinés à traduire ces idées avaient déjà été trouvés. Le moment venu, il ne restait aux auteurs qu'à réunir les éléments épars. Tâchons de discerner d'où venait leur propos.

1. L'Avis commençait par un développement de ce qui avait été brièvement mentionné dans l'oukase du 8 février 1726 sur l'instauration du Conseil : l'impératrice préside le Conseil ; le Conseil jouit uniquement d'un pouvoir consultatif, rien n'en sort qui ne soit signé par l'impératrice, les membres ne contresignent pas les oukases :

Puisque S. M. est la présidente du Conseil secret et parce qu'il y a une raison d'espérer qu'elle y assistera souvent en personne, le Conseil secret ne peut nullement être considéré comme un collège particulier : il sert uniquement à soulager S. M. dans les lourdes tâches de gouvernement; toutes les affaires seront traitées et expédiées sans délai ; la personne veillant à la sécurité de S. M. et au renforcement de l'État ne sera pas seule. [...]

Aucun oukase ne doit être publié avant d'être définitivement arrêté par le Conseil secret, avant que les procès-verbaux ne soient signés, lus à S. M. et confirmés par sa très gracieuse approbation. [...]

Les séances du Conseil secret doivent être consignées de deux façons : sous forme d'un journal, qu'il n'y a pas besoin de signer, et sous forme d'un procès-verbal qui contiendra les résolutions et les arrêts et que les membres signeront ${ }^{108}$.

L'idée que le Conseil secret n'était pas un collège ordinaire avait été soulignée par Osterman dans son mémoire inachevé ( « Les conseillers d'État actuels ne se réunissent pas dans un collège particulier $\left.{ }^{109}\right)$. Que le monarque ne devait pas investir toute sa confiance dans une seule personne, Fick l'avait clairement démontré (« Les affaires d'État sont mieux gérées par six ministres que par un seul »110) et Osterman s'était exprimé dans le même sens («Il est rare qu'un seul ait des connaissances suffisantes pour toutes les affaires $\left.»^{111}\right)$. Fick avait également expliqué que le droit de signature devait appartenir exclusivement au monarque («Ce n’est pas le collège de la Chancellerie, mais le roi qui signe tout $»^{112}$ ) et que tous les rapports entre le monarque et les conseillers devaient obligatoirement être consignés par écrit ${ }^{113}$.

108. Ibid.,p. 93-94.

109. RGADA, f. 370 , op. 1, d. 16, 1.50 ob.

110. ZA, no 414, p. 544.

111. RGADA, f. 370, op. 1, d. 16, 1. 52 .

112. $Z A$, no $^{\circ} 414$, p. 543.

113. Ibid., p. 542-543. 
2. Les fonctions du Conseil suprême secret furent décrites dans l'Avis de façon suivante :

Les affaires du ressort du Conseil secret sont les suivantes: 1) les affaires étrangères ${ }^{114}$;2) toutes les affaires qui relèvent de la très haute décision personnelle de S. M. ${ }^{115}$.

Cette définition correspondait exactement à ce qu'Osterman avait écrit à propos du Cabinet secret ${ }^{116}$, en reprenant les idées de Fick sur le collège de la Chancellerie ${ }^{117}$.

3. En définissant les règles de subordination entre le Conseil suprême secret d'un côté, et le Sénat et les collèges de l'autre, l'auteur de l'Avis écrivait que le contrôle de tous les collèges et des autres institutions administratives appartenait au Conseil secret ${ }^{118}$; que le Sénat et les collèges fonctionneraient d'après leurs règlements ; qu'ils soumettraient cependant au Conseil secret, avec leur avis, les dossiers particulièrement importants, ceux qui ne pouvaient être terminés d'après les lois existantes ou qui dépendaient de la volonté personnelle de S. M. L'Avis précisait que les appels contre les décisions du Sénat et des trois premiers collèges étaient autorisés et devaient être adressés à l'impératrice pour examen au Conseil secret ${ }^{119}$. On reconnaît ici les principes décrits par Fick dans le passage sur les relations entre le collège de la Chancellerie de Charles XI (ou les ministres de Charles XII) et les autres collèges suédois ${ }^{120}$.

4. Fick avait insisté sur le danger que le Sénat (Riksråd) présentait pour le roi de Suède ${ }^{121}$. Les membres du Conseil suprême secret dépouillèrent le Sénat pétrovien de son statut d'institution exécutive supérieure :

Il est désormais impossible de l'appeler [le Sénat] Gouvernant, il faut lui conférer un titre de haut mandataire [vysokopoverenny] ou simplement de haut Sénat ${ }^{122}$.

Osterman avait indiqué dans son mémoire que le chancelier, l'amiral et le feldmaréchal étaient «plus importants»que les autres sénateurs ${ }^{123}$. Le Conseil

114. « Les ministres étrangers relèveront du collège des Affaires étrangères, mais le Collège est tenu de toujours rapporter leurs communications à S. M. lors des séances du Conseil secret », précisait l'auteur de l'A vis dans un autre endroit du même texte.

115. SIRIO, vol. 55, p. 95.

116. RGADA, f. 370 , op. 1, d. 16,1.50.

117. $Z A, \mathrm{n}^{\circ} 414$, p. 542 .

118. SIRIO, vol. 55, p. 96.

119. Ibid., p. 95.

120. $Z A, \mathrm{n}^{\circ} 414$, p. $542-543,545$.

121. Ibid., p. 546-547.

122. SIRIO, vol. 55, p. 96.

123. RGADA, f. 370, op. 1, d. 16, 1. 50-50 ob. 
suprême secret postula l'autonomie de ces officiers, et des services sous leur direction, vis-à-vis du Sénat :

Les trois premiers collèges [ceux des Affaires étrangères, de la Guerre et de l'Amirauté] ne sauront se trouver sous l'autorité du Sénat [... $]^{124}$.

Les affaires diplomatiques, notamment, se trouvèrent ainsi totalement soustraites, même théoriquement, à l'autorité du Sénat pour être placées sous le contrôle immédiat du souverain, conformément à ce que Fick, Osterman et les membres du collège des Affaires étrangères avaient toujours désiré.

5. L'auteur de l'Avis proposait de diviser le Conseil en départements :

Quand S. M. y assistera en personne, elle établira l'ordre du jour d'après sa très gracieuse volonté. Mais quand elle ne daignera pas être présente, il serait préférable de donner à chaque membre un département, ou povyt e $[$ [...].

Il faut que le Conseil secret ait des bureaux et que les domaines de compétence soient partagés, pour que tout soit en bon ordre et que les affaires puissent être expédiées sans confusion et en temps voulu ${ }^{125}$.

Dans ce paragraphe s'entremêlent les voix de Fick et d'Osterman. Fick écrivait que la spécialisation avait été pratiquée au collège de la Chancellerie de Charles XI ${ }^{126}$ et qu'elle fut poussée encore plus loin au Cabinet ministériel de Charles XII ${ }^{127}$. Osterman approuvait cet usage : « Il est bon que les papiers soient partagés et qu'on ait plusieurs départements ${ }^{128}$.

6. En 1724, Osterman avait écrit qu'il était nécessaire de simplifier la procédure au collège des Affaires étrangères pour pouvoir réduire le nombre d'employés, ce qui, par voie de conséquence, permettrait de mieux garder le secret des affaires. Évoquant l'organisation des bureaux du Conseil suprême secret, l'auteur de l'Avis reprenait les mêmes termes :

Puisque ces bureaux doivent servir d'exemple aux autres, il ne faut pas qu'ils fassent autant de paperasserie inutile et que leurs effectifs soient surchargés d'employés surnuméraires. Il faut donc procéder à la création de ces bureaux avec beaucoup de prudence et tout organiser avec la plus grande circonspection, notamment pour garantir le secret des affaires ${ }^{129}$.

7. Dans la Kurze Beschreibung, Fick attirait spécialement l'attention sur le fait que le collège de la Chancellerie portait la responsabilité de ce que tous les documents signés par le roi et publiés en son nom fussent parfaits du point de vue du contenu

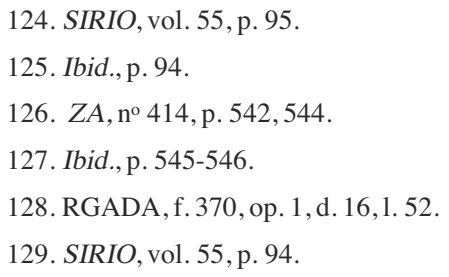


aussi bien que de la forme, pour que les sujets du roi ne doutassent jamais de la justice ni de la pertinence de ses ordres ${ }^{130}$. L'auteur de l'A vis terminait sur la même note :

[...] il ne sied pas [au Conseil suprême secret] d'agir précipitamment, parce que tout ceci est fait au nom auguste de S. M., afin d'en tirer profit pour tout l'Empire de la façon la plus sûre et afin que la plus grande gloire s'en répande dans la nation ${ }^{131}$.

Il paraît évident que l'auteur ou, plutôt, les auteurs de l'A vis puisaient leur inspiration, leurs idées et leurs mots dans les mémoires de Fick et d'Osterman.

\section{Conclusion}

La reconstruction factuelle de la genèse des textes concernant le fonctionnement du gouvernement compte parmi les méthodes relativement peu utilisées en histoire politique russe du XVIII siècle $^{132}$. Les actes normatifs, surtout, sont souvent considérés comme sémantiquement autonomes: une simple lecture d'un texte isolé permettrait à elle seule de comprendre et d'interpréter son sens que l'on suppose en outre unique, adéquat aux idées de l'auteur et invariable dans le temps. En d'autres termes, on croit pouvoir donner une interprétation juste du contenu d'un document normatif sans envisager l'acte de sa création comme un objet historique à part entière. Or, on ne saurait ignorer que les conditions immédiates dans lesquelles un acte normatif donné a été élaboré chargent son contenu de sens implicites, qui peuvent d'ailleurs avoir une influence non négligeable sur les modalités de son application, sur ses effets pratiques directs ou indirects et sur son rayonnement idéologique. L'Avis sur le nouveau Conseil secret qui est institué a été oublié par une partie des historiens du politique et disqualifié comme peu important par l'autre, parce que, précisément, le seul accès au sens de ce texte passe par une historicisation minutieuse de son contenu ${ }^{133}$.

130. ZA, no 414, p. 543.

131. SIRIO, vol. 55 , p. 96.

132. Les études bien connues de Troickij et de Meduševskij sur la Table des rangs ou d'Omel'čenko sur le Nakaz de Catherine II sont quelques-unes des exceptions notables (S. M. Troickij, op. cit.; A. N. Meduševskij, «Tabel' o rangah kak predmet sravnitel'noistoričeskogo issledovanija » (La Table des rangs comme objet d'étude historique comparative), in Issledovanija po istočnikovedeniju istorii SSSR dooktjabr'skogo perioda (Études sur les sources de l'histoire de l'URSS prérévolutionaire), Moscou, 1991 ; O. A. Omel'čenko, "Zakonnaja monarhija » Ekateriny II: prosveščennyj absoljutizm V Rossii («La monarchie légitime» de Catherine II : l'absolutisme éclairé en Russie), Moscou, 1993). La précieuse édition des actes législatifs de Pierre Ir préparée par $\mathrm{N}$. A. Voskresenskij $(Z A)$, où chaque texte est accompagné de tous les brouillons conservés, reste un instrument de travail unique dans son genre (seul le premier volume a été publié ; les deux autres dorment, paraît-t-il, dans les archives de l'Institut d'histoire russe de l'Académie des sciences, à Moscou).

133. D. N. Šanskij, l'un des rares historiens à mentionner l'Avis, limite son analyse à une conclusion que ce texte « formule des principes généraux de l'autonomie et des pleins pouvoirs [du Conseil] plutôt qu'il ne les restreint » et qu'il ne peut donc être considéré comme un règlement 
Le fait de suivre l'élaboration de l'Avis jusqu'à sa source première a permis de montrer que le Conseil suprême secret de 1726 fut plus qu'un gouvernement provisoire, formé par les bojare aux côtés d'un souverain inapte, et l'Avis plus qu'une tentative maladroite d'introduire un peu d'ordre dans le fonctionnement de ce gouvernement. La création du Conseil et la rédaction de l'Avis sont le point culminant atteint par une vague de la pensée politique, soulevée par la déchéance d'un ancien modèle idéologique qui avait soutenu, jusqu'à la fỉn du XVII ${ }^{\mathrm{e}}$ siècle, la pratique de conseil ritualisé entre le tsar et ses serviteurs de confiance. Le Conseil suprême secret en sa forme initiale fut une incarnation du modèle élaboré par les « projeteurs » politiques du règne de Pierre I ${ }^{e r}$, dont les sympathies allaient vers une conception occidentale contemporaine du conseil: «laïque (relativement déchargée de parallèles entre le pouvoir du tsar et le pouvoir divin), étroite et institutionnelle. L'actualisation de ce modèle en 1726 marqua un temps fort dans l'évolution absolutiste de la monarchie russe ${ }^{134}$.

On peut supposer que, malgré la médiocrité de l'effet pratique des dispositions contenues dans l'Avis, elles entrèrent durablement dans l'outillage mental de l'élite politique russe. Il serait intéressant, par exemple, de reprendre sous cet éclairage l'analyse des célèbres «projets oligarchiques », rédigés en 1730 par les membres du Conseil suprême secret et en 1762 par Nikita Panin ${ }^{135}$. Le projet de $1762^{136}$, notamment, a souvent été interprété comme une expression des idées constitutionnelles que Panin aurait conçues lors de son séjour à Stockholm en tant qu'ambassadeur de l'impératrice. Pourtant, Panin pouvait avoir d'autres sources d'inspiration que la Suède des années 1750. On n'a jamais tenté de replacer son projet de Conseil impérial dans le paradigme des idées politiques russes, dont l'Avis reste un des témoignages les plus importants. Pour notre part, nous pensons que le projet de Panin est tributaire des idées que Fick et Osterman s'étaient donné tant de peine à

(D. N. Sanskij, « K harakteristike vysših gosudarstvennyh učreždenij Rossii XVIII veka (20e_ 60 gg.) » (À propos de la nature des institutions d'État suprêmes en Russie dans les années 1720-1760), in N. B. Golikova, ed., Gosudarstvennye učreždenija Rossii XVI-XVIII VV. (Les insitutions d'État de Russie, XVI-XVIII s.), Moscou, Moskovskij gosudarstvennyj universitet, 1991,p. 122.

134. Voir un stimulant article de Šanskij qui développe l'idée que, à partir du règne de Pierre Irer, « la mentalité politique au sommet de la classe dominante se détourna [...] de la tradition de la monarchie avec une Duma et une aristocratie des boyards » ; pour Šanskij, le Conseil suprême secret est « un organe proprement absolutiste » (D. N. Šanskij, art. cit., p. 122-123).

135. Les documents de 1730, rédigés lors de l'avènement de l'impératrice Anna Ivanovna, ou le projet de Conseil impérial, écrit à l'attention de Catherine II en 1762 et attribué à Nikita Panin, sont interprétés, aujourd'hui encore, comme des «projets oligarchiques», des «tentatives de limitation du pouvoir du tsar» et même, concernant les Conditions de 1730, comme le début de la «tradition du droit constitutionnel en Russie » (S. Bertolissi, A. N. Saharov, A. N. Meduševskij, eds., Konstitucionnye proekty $v$ Rossii, XVIII-načalo XX v. (Les projets constitutionnels en Russie, $X V I I I^{\mathrm{e}}$-début $X X^{\mathrm{e}}$ siècle), Moscou, Institut Rossijskoj istorii Rossijskoj Akademii Nauk, 2000, Introduction, p. 3).

136. Le texte de Panin est publié dans SIRIO, 1871, vol. 7, p. 202-217. Voir une traduction anglaise, partielle, dans Marc Raeff, ed., Plans for political reform in Imperial Russia, 17301905, Englewood Cliffs, NJ, Prentice Hall, 1966, p. 54-68. 
propager et probablement de leurs écrits ${ }^{137}$. La vérification de cette hypothèse éclairerait une des questions centrales de l'historiographie russe, celle de $l^{\prime}$ « opposition aristocratique » à l'absolutisme au XVIII' siècle ${ }^{138}$.

Centre d'études du monde russe, soviétique et post-soviétique

EHESS

54, boulevard Raspail

75006 Paris

lecerf@gage.polytechnique.fr

137. Nous partageons une hypothèse de David Ransel qui voit une parenté typologique entre le projet de Conseil impérial de 1762 et les conseils, ou projets de conseils, antérieurs. Selon lui, Panin demeura fidèle à la logique des concepteurs du Conseil suprême secret. Ransel a été le principal critique de l'interprétation « constitutionnelle » des idées de Panin (D. Ransel, The politics of Catherinian Russia. The Panin party, New Haven, CT, Yale University Press, 1975, p. 134-138).

138. La thèse de l'opposition aristocratique, voire nobiliaire, a été longtemps dominante dans l'historiographie du XVIII ${ }^{\mathrm{e}}$ siècle russe. Voir sa critique et une vision globale de la Russie du $\mathrm{XVIII}^{\mathrm{e}}$ siècle dirigée par une «classe gouvernante » hétérogène mais largement consensuelle dans les travaux de John LeDonne, Ruling Russia. Politics and administration in the Age of Absolutism, 1762-1796, Princeton, NJ, Princeton University Press, 1984, p. 3-22 ; id., Absolutism and ruling class. The formation of the Russian political order, 1700-1825, New York, Oxford University Press, 1991, p. vii-xii, 3-60 et passim. 